\title{
The Human T Cell Receptor Alpha Joining (TRAJ) Genes
}

\author{
Dominique Scaviner Marie-Paule Lefranc \\ Laboratoire d'ImmunoGénétique Moléculaire, CNRS, Université Montpellier II, Montpellier, France
}

\section{Key Words}

Human genes · IMGT · T cell receptor · Alpha joining genes

\footnotetext{
Abstract

'Human $T$ cell Receptor Alpha Joining Genes', the 9th report of the 'IMGT Locus in Focus' section, comprises 3 tables: (1) 'Human germline TRAJ genes'; (2) 'Human TRAJ allele table'; and (3) 'Nucleotide and protein displays of the human TRAJ alleles (overview)'. These tables are available on the IMGT Marie-Paule page from IMGT, the international ImMunoGeneTics database (http:/ /imgt.cines.fr:8104) created in 1989 by Marie-Paule Lefranc, Université Montpellier II, CNRS, France.
}

Copyright $\odot 2000$ S. Karger AG, Basel

\section{Introduction}

'Human $\mathrm{T}$ cell Receptor Alpha Joining Genes' is the 9th report of the 'IMGT Locus in Focus' section launched in the April 1998 issue of Experimental and Clinical Immunogenetics [1]. We have previously reported the complete repertoire of the human germline IGH, IGK and IGL genes [2-6], and that of the germline human TRBV [7] and TRAV [8] genes. This 9th report on the human $\mathrm{T}$ cell receptor alpha joining genes comprises 3 tables: (1) 'Human germline TRAJ genes'; (2) 'Human TRAJ allele table'; (3) 'Nucleotide and protein displays of the human TRAJ alleles (overview)'. These tables are available on the IMGT Marie-Paule page from IMGT, the international ImMunoGeneTics database (http://imgt.cines.fr:8104) created in 1989 by Marie-Paule Lefranc, Université Montpellier II, CNRS, France [9, 10]. Descriptions of functionality (functional, open reading frame, pseudogene) and of mutations are according to the IMGT Scientific chart [9, 10], available on the IMGT Marie-Paule page.

Prof. Marie-Paule Lefranc, Université Montpellier II

Lab. d'ImmunoGénétique Moléculaire, LIGM, UPR CNRS 1142, IGH

141, rue de la Cardonille, F-34396 Montpellier Cedex 5 (France)

Tel. +33499619965, Fax +33499619901

E-Mail lefranc@ligm.igh.cnrs.fr, IMGT: http://imgt.cines.fr:8104
Fax +4161306 1234

E-Mail karger@karger.ch Accessible online at:

www.karger.com www.karger.com/journals/eci 
Table 1. Human germline TRAJ genes

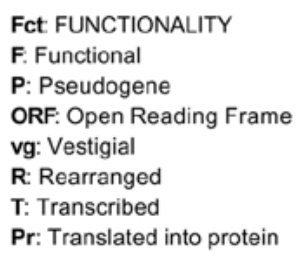

"+" or "-" indicates if the gene sequences have been found (+) or not been found (-) rearranged (R), transcribed (T), and/or translated into protein (Pr).

Arbitrarily that information is shown on the first line of each gene when the data have been confirmed by several studies. Note that AE000661 encompasses M94081 from TRAJ61 to TRAJ46, and AE000662 encompasses M94081 from TRAJ45 to TRAJ1. Sequencing and/or typing errors initially detected by IMGT in M94081 sequence (TRAJ15, TRAJ36, TRAJ37) have been corrected with the author's authorization (B.K.) in EMBL (11/97). These errors have not yet been corrected in AE000662 sequence.

\begin{tabular}{|c|c|c|c|c|c|c|c|c|c|}
\hline TRAJ name & Fct & $R^{*} \mid \mathbf{T}$ & $T^{*} \mathbf{F}$ & $P^{*}$ & \begin{tabular}{l|} 
Reference \\
sequences
\end{tabular} & $\begin{array}{l}\text { Accession } \\
\text { numbers }\end{array}$ & $\begin{array}{l}\text { Position in } \\
\text { M94081 (a) }\end{array}$ & $\begin{array}{l}\text { Position in } \\
\text { M94081 (b) }\end{array}$ & Sequences from the literature \\
\hline TRAJ1 & ORF(1) & & $-{ }_{-1}$ & - & & $\mathrm{X} 02884$ [1] & $84789-86510$ & $85235-85296$ & J1[M94081][7], [AE000662][8] \\
\hline TRAJ2 & ORF(2) & & & & & $\mathrm{X02884[1]}$ & $83858-84788$ & $84269-84334$ & $\begin{array}{l}\text { JaSP[X05003][2], JaSP[M14862][2], } \\
\text { J2[M94081][7], [AE000662][8] }\end{array}$ \\
\hline TRAJ3 & $\mathrm{F}$ & + & + & & & X02884 [1] & $82422-83857$ & $83376-83337$ & \begin{tabular}{|l} 
JaSP[X05003][2], JaSP[M14862][2], \\
J3[M94081][7], [AE000662][8]
\end{tabular} \\
\hline TRAJ4 & $\mathrm{F}$ & + & + & & $\mathrm{J4}$ & M94081 [7] & $80952-82421$ & \begin{tabular}{|l|}
$82396-82458$ \\
\end{tabular} & [AE000662][8] \\
\hline TRAJ5 & $\mathrm{F}$ & +1. & + & & J5 & M94081 [7] & 79892-80951 & $80444-80503$ & [AE000662][8] \\
\hline TRAJ6 & $\mathrm{F}$ & ++ & + & & JA5 & M16747 [4] & \begin{tabular}{|l|}
$78579-79891$ \\
\end{tabular} & \begin{tabular}{|l|}
$79270-79331$ \\
\end{tabular} & J6[M94081][7], [AE000662][8] \\
\hline TRAJ7 & $\mathrm{F}$ & + & + & & J7 & M94081 [7] & \begin{tabular}{|l|}
$77118-78578$ \\
\end{tabular} & 77821-77879 & {$[\mathrm{AE} 000662][8]$} \\
\hline TRAJ8 & $\mathrm{F}$ & + & + & & J8 & M94081 [7] & \begin{tabular}{|l|}
$76086-77117$ \\
\end{tabular} & \begin{tabular}{|l|}
$76346-76405$ \\
\end{tabular} & [AE000662][8] \\
\hline TRAJ9 & $\mathrm{F}$ & + & + & & J9 & M94081 [7] & \begin{tabular}{|l|}
$74764-76085$ \\
\end{tabular} & \begin{tabular}{|l|}
$75756-75816$ \\
\end{tabular} & [AE000662][8] \\
\hline TRAJ10 & $\mathrm{F}$ & + & + & & $\mathrm{J} 10$ & M94081 [7] & \begin{tabular}{|l|l|}
$73237-74763$ \\
\end{tabular} & \begin{tabular}{|l|}
$73699-73762$ \\
\end{tabular} & [AE000662][8] \\
\hline TRAJ11 & $\mathrm{F}$ & ++ & + & & J11 & M94081 [7] & \begin{tabular}{|l|}
$72459-73236$ \\
\end{tabular} & \begin{tabular}{|l|}
$72706-72765$ \\
\end{tabular} & [AE000662][8] \\
\hline TRAJ12 & $\mathrm{F}$ & + & + & & & $\mathrm{X} 02885[1]$ & \begin{tabular}{|l|}
$71747-72458$ \\
\end{tabular} & \begin{tabular}{|l|}
$72143-72202$ \\
\end{tabular} & J12[M94081][7], [AE000662][8] \\
\hline TRAJ13 & $\mathrm{F}$ & +1 & + & & J13 & M94081 [7] & 70936-71746 & $71280-71342$ & [AE000662][8] \\
\hline TRAJ14 & $\mathrm{F}$ & & & & J14 & M94081 [7] & 70218-70935 & \begin{tabular}{|l|}
$70532-70583$ \\
\end{tabular} & [AE000662][8] \\
\hline \multirow[t]{2}{*}{ TRAJ15 } & $\mathrm{F}$ & ++ & + & & JaS & $\mathrm{X} 05775[5]$ & & & \\
\hline & $\mathrm{F}$ & ++ & + & & J15 & M94081 [7] & 69322-70217 & 69836-69895 & [AE000662][8] \\
\hline TRAJ16 & $F(3)$ & ++ & + & & J16 & M94081 [7] & 67939-69321 & 68743-68802 & [AE000662][8] \\
\hline TRAJ17 & $\mathrm{F}$ & ++ & + & & JaLv & $\mathrm{X} 05773[5]$ & \begin{tabular}{|l|}
$66507-67938$ \\
\end{tabular} & \begin{tabular}{|l|}
$67068-67130$ \\
\end{tabular} & J17[M94081][7], [AE000662][8] \\
\hline TRAJ18 & \begin{tabular}{|l|}
$\mathrm{F}$ \\
\end{tabular} & + & + & & J18 & M94081 [7] & \begin{tabular}{|l|}
$65715-66506$ \\
\end{tabular} & \begin{tabular}{|l|}
$65876-65941$ \\
\end{tabular} & {$[\mathrm{AE} 000662][8]$} \\
\hline TRAJ19 & ORF(4) & & & & J19 & M94081 [7] & 65051-65714 & \begin{tabular}{|l|}
$65489-65548$ \\
\end{tabular} & [AE000662][8] \\
\hline TRAJ20 & \begin{tabular}{|l|}
$\mathrm{F}$ \\
\end{tabular} & + & + & - & $\mathrm{J} 20$ & M94081 [7] & 64222-65050 & \begin{tabular}{|l|}
$64552-64608$ \\
\end{tabular} & [AE000662][8] \\
\hline TRAJ21 & $\mathrm{F}$ & + & + & & J21 & M94081 [7] & 63083-64221 & 63829-63883 & [AE000662][8] \\
\hline TRAJ22 & $\mathrm{F}$ & + & + & & J22 & $\mathrm{X} 02886[1]$ & \begin{tabular}{|l|}
$61495-63082$ \\
\end{tabular} & $62272-62334$ & J22[M94081][7], [AE000662][8] \\
\hline TRAJ23 & $\mathrm{F}$ & +1 & + & & $\mathrm{J} 23$ & M94081 [7] & \begin{tabular}{|l|l}
$60460-61494$ \\
\end{tabular} & $60650-60712$ & [AE000662][8] \\
\hline \multirow[t]{2}{*}{ TRAJ24 } & $\mathrm{F}$ & & & & & $\mathrm{X} 02887[1]$ & & & \\
\hline & $\mathrm{F}$ & & & & & M94081 [7] & 59657-60459 & $60203-60265$ & {$[\mathrm{AE} 000662][8]$} \\
\hline TRAJ25 & ORF(5) & & & & & $\mathrm{X} 02888[1]$ & 58895-59656 & 59046-59105 & J25[M94081][7], [AE000662][8] \\
\hline TRAJ26 & \begin{tabular}{|l|}
$\mathrm{F}$ \\
\end{tabular} & + & + & & J26 & M94081 [7] & \begin{tabular}{|l|l}
$57625-58894$ \\
\end{tabular} & \begin{tabular}{|l|}
$58680-58739$ \\
\end{tabular} & [AE000662][8] \\
\hline TRAJ27 & $\mathrm{F}$ & ++ & + & - & J27 & M94081 [7] & 56217-57624 & \begin{tabular}{|l|}
$56507-56565$ \\
\end{tabular} & [AE000662][8] \\
\hline TRAJ28 & $\mathrm{F}$ & ++ & + & - & J28 & M94081 [7] & \begin{tabular}{|l|}
$55049-56216$ \\
\end{tabular} & \begin{tabular}{|l|}
$55857-55922$ \\
\end{tabular} & [AE000662][8] \\
\hline TRAJ29 & $\mathrm{F}$ & ++ & + & & & $\mathrm{X} 02889[1]$ & \begin{tabular}{|l|}
$53664-55048$ \\
\end{tabular} & \begin{tabular}{|l|}
$54177-54236$ \\
\end{tabular} & J29[M94081][7], [AE000662][8] \\
\hline TRAJ30 & $\mathrm{F}$ & ++ & + & & $\mathrm{J} 30$ & M94081 [7] & \begin{tabular}{|l|l}
$52179-53663$ \\
\end{tabular} & \begin{tabular}{|l|}
$53090-53146$ \\
\end{tabular} & [AE000662][8] \\
\hline TRAJ31 & $\mathrm{F}$ & + & + & & & M14905 [3] & $50429-52178$ & $53090-53146$ & J31[M94081][7], [AE000662][8] \\
\hline TRAJ32 & $\mathrm{F}$ & + & + & & J32 & M94081 [7] & 49243-50248 & $49581-49646$ & {$[\mathrm{AE} 000662][8]$} \\
\hline TRAJ33 & $\mathrm{F}$ & + & + & & J33 & M94081 [7] & 48406-49242 & \begin{tabular}{|l|}
$48843-48899$ \\
\end{tabular} & [AE000662][8] \\
\hline TRAJ34 & $\mathrm{F}$ & + & ++ & & JaAB & M35622 [6] & 47428-48405 & \begin{tabular}{|l|}
$47907-47964$ \\
\end{tabular} & J34[M94081][7], [AE000662][8] \\
\hline TRAJ35 & \begin{tabular}{|l|}
$\operatorname{ORF}(6)$ \\
\end{tabular} & 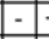 & + & - & J35 & M94081 [7] & 46151-47427 & \begin{tabular}{|l|}
$46885-46943$ \\
\end{tabular} & [AE000662][8] \\
\hline TRAJ36 & $\mathrm{F}$ & + & & & J36 & M94081 [7] & \begin{tabular}{|l|}
$44705-46150$ \\
\end{tabular} & \begin{tabular}{|l|}
$45353-45411$ \\
\end{tabular} & {$[\mathrm{AE} 000662][8]$} \\
\hline TRAJ37 & $\mathrm{F}$ & + & + & & J37 & M94081 [7] & $43204-44704$ & $43993-44054$ & [AE000662][8] \\
\hline
\end{tabular}


Table 1 (continued)

\begin{tabular}{|c|c|c|c|c|c|c|c|c|c|}
\hline TRAJ name & Fct & $\mathbf{R}^{*}$ & $T^{*}$ & $\mathrm{P}^{*}$ & \begin{tabular}{|l|} 
Reference \\
sequences
\end{tabular} & $\begin{array}{l}\text { Accession } \\
\text { numbers }\end{array}$ & $\begin{array}{l}\text { Position in } \\
\text { M94081 (a) }\end{array}$ & $\begin{array}{l}\text { Position in } \\
\text { M94081 (b) }\end{array}$ & Sequences from the literature \\
\hline TRAJ38 & $\mathrm{F}$ & + & + & & J38 & M94081 [7] & $42130-43203$ & $42473-42534$ & [AE000662][8] \\
\hline TRAJ39 & $\mathrm{F}$ & + & + & & J39 & M94081 [7] & $40917-42129$ & 41843-41905 & [AE000662][8] \\
\hline TRAJ40 & $\mathrm{F}$ & + & + & & JaAA & M35620 [6] & $38946-40916$ & $39930-39990$ & J40[M94081][7], [AE000662][8] \\
\hline TRAJ41 & $\mathrm{F}$ & + & + & & $\mathrm{J} 41$ & M94081 [7] & $37548-38945$ & $37900-37961$ & {$[\mathrm{AE} 000662][8]$} \\
\hline TRAJ42 & $\mathrm{F}$ & + & + & & $\mathrm{J} 42$ & M94081 [7] & $36669-37547$ & $37130-37195$ & [AE000662][8] \\
\hline TRAJ43 & $\mathrm{F}$ & + & & & $\mathrm{J} 43$ & M94081 [7] & $35641-36668$ & $36154-36207$ & [AE000662][8] \\
\hline TRAJ44 & $\mathrm{F}$ & + & + & & JaRP & M35619 [6] & $34650-35640$ & $35064-35126$ & J44[M94081][7], [AE000662][8] \\
\hline TRAJ45 & $\mathrm{F}$ & + & + & & $\mathrm{J} 45$ & M94081 [7] & $33940-34649$ & $34169-34234$ & {$[\mathrm{AE} 000662][8]$} \\
\hline TRAJ46 & $\mathrm{F}$ & & & & $\mathrm{J} 46$ & M94081 [7] & $33400-33939$ & $33647-33709$ & [AE000661][8] \\
\hline TRAJ47 & $\mathrm{F}$ & + & + & & $\mathrm{J} 47$ & M94081 [7] & 31948-33399 & $33096-33152$ & [AE000661][8] \\
\hline TRAJ48 & $\mathrm{F}$ & + & + & & $\mathrm{J} 48$ & M94081 [7] & $30264-31947$ & $30737-30799$ & [AE000661][8] \\
\hline TRAJ49 & $\mathrm{F}$ & + & + & & J49 & M94081 [7] & $29317-30263$ & $29734-29789$ & {$[\mathrm{AE} 000661][8]$} \\
\hline TRAJ50 & $\mathrm{F}$ & + & & & $\mathrm{J} 50$ & M94081 [7] & $28166-29316$ & $28839-28898$ & [AE000661][8] \\
\hline TRAJ51 & $\mathrm{P}(7)$ & & - & - & J51 & M94081 [7] & $27017-28165$ & $27429-27491$ & [AE000661][8] \\
\hline TRAJ52 & $\mathrm{F}$ & + & + & & $\mathrm{J} 52$ & M94081 [7] & $24846-27016$ & $26474-26542$ & [AE000661][8] \\
\hline TRAJ53 & $\mathrm{F}$ & + & + & & J53 & M94081 [7] & 22923-24845 & $23251-23316$ & [AE000661][8] \\
\hline TRAJ54 & $\mathrm{F}$ & + & + & - & J54 & M94081 [7] & $22328-22922$ & $22534-22593$ & [AE000661][8] \\
\hline TRAJ55 & $\mathrm{P}(7)$ & & & & J55 & M94081 [7] & 19887-22327 & $21944-22000$ & [AE000661][8] \\
\hline TRAJ56 & $\mathrm{F}$ & + & + & & J56 & M94081 [7] & 19475-19886 & \begin{tabular}{|l|}
$19768-19829$ \\
\end{tabular} & [AE000661][8] \\
\hline TRAJ57 & $\mathrm{F}$ & + & + & & J57 & M94081 [7] & 18568-19474 & \begin{tabular}{|l}
$19119-19181$ \\
\end{tabular} & [AE000661][8] \\
\hline TRAJ58 & ORF(8) & + & + & & J58 & M94081 [7] & $17405-18467$ & $17954-18016$ & {$[$ AE000661][8] } \\
\hline TRAJ59 & ORF(9) & + & - & - & J59 & M94081 [7] & 16704-17404 & $16801-16854$ & {$[$ AE000661][8] } \\
\hline TRAJ60 & $\mathrm{P}(7)$ & + & & & $\mathrm{J} 60$ & M94081 [7] & 16089-16703 & \begin{tabular}{|l|}
$16554-16610$ \\
\end{tabular} & [AE000661][8] \\
\hline TRAJ61 & ORF(9) & + & & & J61 & M94081 [7] & 12582-16088 & $15564-15623$ & [AE000661][8] \\
\hline
\end{tabular}

(a) Arbitrary limits of the DNA sequence assigned to the different TRA J-SEGMENTs, in M94081.

(b) Limits of the J-REGION.

IMGT notes:

(1) Non canonical J-HEPTAMER: GGGCATG instead of CACTGTG, and non canonical J-NONAMER: ATTCTGT instead of GGTTTTTGT.

(2) Non canonical J-HEPTAMER: TACGGTA instead of CACTGTG.

(3) Note in the J-REGION: Phe-Ala-X-Gly instead of Phe-Gly-X-Gly.

(4) 7 nucleotides instead of 12 in J-SPACER.

(5) Non canonical J-HEPTAMER: CACTATG instead of CACTGTG.

(6) Non canonical J-REGION: Cys-Gly-X-Gly instead of Phe-Gly-X-Gly.

(7) STOP-CODON in J-REGION.

(8) Note that the first codon of the germline J-REGION is a STOP-CODON which may disappear during rearrangements.

(9) Defective DONOR_SPLICE.

- These information have been obtained from the following publications:

Roman-Roman, S. et al., Eur. J. Immunol., 21, 927-933 (1991).

Giachino, C. et al., J. Immunol. Res., 4, 99-105 (1992).

Moss, P.A.H. et al., Eur. J. Immunol., 23, 1153-1159 (1993).

Roman-Roman, S. et al., Molec. Immun., 30, 423-431 (1993).

References:

Koop, B.F.et al., Genomics, 19, 478-493 (1994).

[1] Yoshikai, Y. et al., Nature, 316, 837-839 (1985).

[2] Baer, R.J. et al., Mol. Biol. Med., 3, 265-277 (1986).

[3] Finger, L.R. et al., Science, 234, 982-985 (1986).

[4] Baer, R.J. et al., Cell, 50, 97-105 (1987).

[5] Mengle-Gaw, L. et al., EMBO J., 6, 2273-2280 (1987)

[6] Baer, R.J. et al., EMBO J., 7, 1661-1668 (1988).

[7] Koop, B.F. et al., Genomics, 19, 478-493 (1994).

[8] Boysen, C. et al., unpublished.

Author: Dominique Scaviner imgt@ligm.igh.cnrs.fr

Created: $27 / 10 / 97$

Last updated: 18/01/99 
Table 2. Human TRAJ allele table

Fct: FUNCTIONALITY

F: Functional

P: Pseudogene

ORF: Open Reading Frame

vg: Vestigial

IMGT numbering and description of alleles for germline J-REGIONs start with the first nucleotide of the first codon.

The accession number of a reference sequence is given for each allele.

\begin{tabular}{|c|c|c|c|c|c|}
\hline $\begin{array}{l}\text { TRAJ } \\
\text { name }\end{array}$ & Fct & $\begin{array}{l}\text { TRAJ } \\
\text { allele name }\end{array}$ & $\begin{array}{c}\text { Accession } \\
\text { number }\end{array}$ & $\begin{array}{l}\text { Confirmed by } \\
\text { genetics } \\
\text { and/or data }\end{array}$ & Description of mutations \\
\hline TRAJ1 & ORF & TRAJ1*01 $^{*}$ & $\mathrm{X} 02884$ & + & \\
\hline TRAJ2 & ORF & TRAJ2*01 & $\mathrm{X02884}$ & + & \\
\hline TRAJ3 & $\mathrm{F}$ & TRAJ3*01 & $\mathrm{X} 02884$ & + & \\
\hline TRAJ4 & $\mathrm{F}$ & TRAJ4*01 & M94081 & & \\
\hline TRAJ5 & $\mathrm{F}$ & TRAJ5*01 & M94081 & & \\
\hline TRAJ6 & $\mathrm{F}$ & TRAJ6*01 & M16747 & + & \\
\hline TRAJ7 & $\mathrm{F}$ & TRAJ7*01 & M94081 & & \\
\hline TRAJ8 & $\mathrm{F}$ & TRAJ8*01 & M94081 & & \\
\hline TRAJ9 & $\mathrm{F}$ & TRAJ9*01 & M94081 & & \\
\hline TRAJ10 & $\mathrm{F}$ & TRAJ10*01 & M94081 & & \\
\hline TRAJ11 & $\mathrm{F}$ & TRAJ11*01 & M94081 & & \\
\hline TRAJ12 & $\mathrm{F}$ & TRAJ12*01 & X02885 & + & \\
\hline TRAJ13 & $\mathrm{F}$ & TRAJ13*01 & M94081 & & \\
\hline TRAJ14 & $\mathrm{F}$ & TRAJ14*01 & M94081 & & \\
\hline \multirow[t]{2}{*}{ TRAJ15 } & $\mathrm{F}$ & TRAJ15*01 & X05775 & & \begin{tabular}{|lll|l}
$\mathrm{a} 40, \mathrm{C} 41$ & ,T14 & $\mathrm{t} 43$
\end{tabular} \\
\hline & $\mathrm{F}$ & TRAJ15*02 & M94081 & & $|\mathrm{a} 40>\mathrm{C}, \mathrm{C} 41>\mathrm{a}, \mathrm{T} 14>\mathrm{H}| \mathrm{t} 43>\mathrm{C} \mid$ \\
\hline TRAJ16 & $\mathrm{F}$ & TRAJ16*01 & M94081 & & \\
\hline TRAJ17 & $\mathrm{F}$ & TRAJ17*01 & $\mathrm{X05773}$ & + & \\
\hline TRAJ18 & $\mathrm{F}$ & TRAJ18*01 & M94081 & & \\
\hline TRAJ19 & ORF & TRAJ19*01 & M94081 & & \\
\hline TRAJ20 & $\mathrm{F}$ & TRAJ20*01 & M94081 & & \\
\hline TRAJ21 & $\mathrm{F}$ & TRAJ21*01 & M94081 & & \\
\hline TRAJ22 & $\mathrm{F}$ & TRAJ22*01 & $\mathrm{X} 02886$ & + & \\
\hline TRAJ23 & $\mathrm{F}$ & TRAJ23*01 & M94081 & & \\
\hline \multirow[t]{2}{*}{ TRAJ24 } & $\mathrm{F}$ & TRAJ24*01 & $\mathrm{X} 02887$ & & \begin{tabular}{|ll|ll|} 
C24 & ,F8 & g25 & ,E9
\end{tabular} \\
\hline & $\mathrm{F}$ & TRAJ24*02 & M94081 & & $\mathrm{C} 24>\mathrm{g}, \mathrm{F} 8>\mathrm{L}|\mathrm{g} 25>\mathrm{C}, \mathrm{E} 9>\mathrm{Q}|$ \\
\hline TRAJ25 & ORF & TRAJ25*01 & $\mathrm{X} 02888$ & + & \\
\hline TRAJ26 & $\mathrm{F}$ & TRAJ26*01 & M94081 & & \\
\hline TRAJ27 & $\mathrm{F}$ & TRAJ27 $7^{*} 01$ & M94081 & & \\
\hline TRAJ28 & $\mathrm{F}$ & TRAJ28*01 & M94081 & & \\
\hline TRAJ29 & $\mathrm{F}$ & TRAJ29*01 & X02889 & + & \\
\hline TRAJ30 & $\mathrm{F}$ & TRAJ30*01 & M94081 & & \\
\hline TRAJ31 & $\mathrm{F}$ & TRAJ31*01 & M14905 & + & \\
\hline TRAJ32 & $\mathrm{F}$ & TRAJ32*01 & M94081 & & \\
\hline TRAJ33 & $\mathrm{F}$ & TRAJ33*01 & M94081 & & \\
\hline TRAJ34 & $\mathrm{F}$ & TRAJ34*01 & M35622 & + & \\
\hline TRAJ35 & ORF & TRAJ35*01 & M94081 & & \\
\hline
\end{tabular}


Table 2 (continued)

\begin{tabular}{|c|c|c|c|c|c|}
\hline $\begin{array}{l}\text { TRAJ } \\
\text { name }\end{array}$ & Fct & $\begin{array}{l}\text { TRAJ } \\
\text { allele name }\end{array}$ & $\begin{array}{c}\text { Accession } \\
\text { number }\end{array}$ & $\begin{array}{l}\text { Confirmed by } \\
\text { genetics } \\
\text { and/or data }\end{array}$ & Description of mutations \\
\hline TRAJ36 & $\mathrm{F}$ & TRAJ36*01 & M94081 & & \\
\hline TRAJ37 & $\mathrm{F}$ & TRAJ $37^{\star} 01$ & M94081 & & \\
\hline TRAJ38 & $\mathrm{F}$ & TRAJ38*01 & M94081 & & \\
\hline TRAJ39 & $\mathrm{F}$ & TRAJ39*01 & M94081 & & \\
\hline TRAJ40 & $\mathrm{F}$ & TRAJ40*01 & M35620 & + & \\
\hline TRAJ41 & $\mathrm{F}$ & TRAJ41*01 & M94081 & & \\
\hline TRAJ42 & $\mathrm{F}$ & TRAJ42*01 & M94081 & & \\
\hline TRAJ43 & $\mathrm{F}$ & TRAJ43*01 & M94081 & & \\
\hline TRAJ44 & $\mathrm{F}$ & TRAJ44*01 & M35619 & + & \\
\hline TRAJ45 & $\mathrm{F}$ & TRAJ45*01 & M94081 & & \\
\hline TRAJ46 & $\mathrm{F}$ & TRAJ46*01 & M94081 & & \\
\hline TRAJ47 & $\mathrm{F}$ & TRAJ47*01 & M94081 & & \\
\hline TRAJ48 & $\mathrm{F}$ & TRAJ48*01 & M94081 & & \\
\hline TRAJ49 & $\mathrm{F}$ & TRAJ49*01 & M94081 & & \\
\hline TRAJ50 & $\mathrm{F}$ & TRAJ50*01 & M94081 & & \\
\hline TRAJ52 & $\mathrm{F}$ & TRAJ52*01 & M94081 & & \\
\hline TRAJ53 & $\mathrm{F}$ & TRAJ53*01 & M94081 & & \\
\hline TRAJ54 & $\mathrm{F}$ & TRAJ54*01 & M94081 & & \\
\hline TRAJ56 & $\mathrm{F}$ & TRAJ56*01 & M94081 & & \\
\hline TRAJ57 & $\mathrm{F}$ & TRAJ57*01 & M94081 & & \\
\hline TRAJ58 & ORF & TRAJ58*01 & M94081 & & \\
\hline TRAJ59 & ORF & TRAJ59*01 & M94081 & & \\
\hline TRAJ61 & ORF & TRAJ61*01 & M94081 & & \\
\hline
\end{tabular}

Author: Dominique Scaviner imgt@ligm.igh.cnrs.fr

Created: 18/01/99 


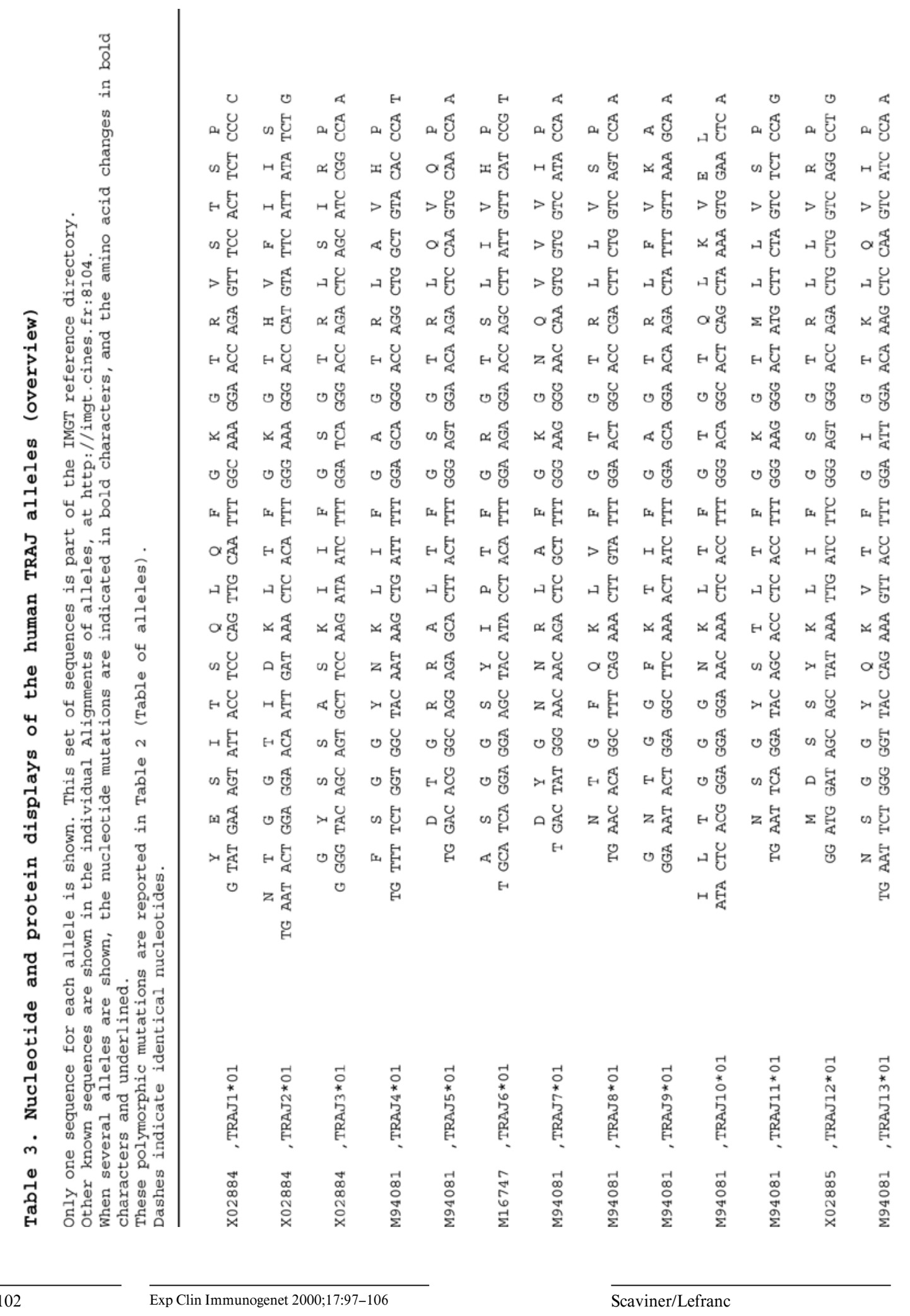




\begin{tabular}{|c|c|c|c|c|c|c|c|c|c|c|c|c|c|c|c|c|}
\hline $\begin{array}{l}0 \\
H\end{array}$ & os & & 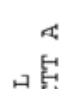 & 象 & $\begin{array}{r}0 \\
\text { تص }\end{array}$ & $\begin{array}{ll}a & 4 \\
0 & 0\end{array}$ & $\begin{array}{l}4 \\
0 \\
4\end{array}$ & 4 & $\begin{array}{r}0 \\
\text { 苞 }\end{array}$ & 娄 & 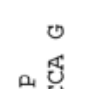 & ' & f & $\begin{array}{r}H \\
U \\
0\end{array}$ & 蛋 & $\begin{array}{rl}4 & 4 \\
a_{1} & 0\end{array}$ \\
\hline 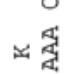 & 心萭 & 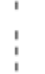 & 口画 & ×乐 & 3 & H莧 & 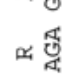 & $\therefore$ & 口 & $\times$ & HU & & $\therefore$ & 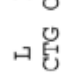 & $\times$ & H \\
\hline & $>$ & 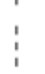 & $>$ > & $>$ 咆 & $>$ > & $>$ 蒠 & $>$ 眮 & $>$ 画 & $>\mathrm{E}$ & $>$ & $>$ 苞 & 7 & $>$ & $>$ > & $>$ 品 & $>$ 苞 \\
\hline & co 䁬 & $\vdots$ & 듈 & ה & H压 & 口艇 & 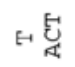 & 吅菜 & $H \tilde{q}$ & 为莺 & $>$ 昀 & : & & is & B若 & os \\
\hline & 口乐 & i & ك. & $>$ 笣 & & x 宊 & $>$ 泉 & & نِ & બ & $>$ 鸹 & & & & 믈 & $\rightarrow$ \\
\hline & $\mathrm{H}$ & & $\Sigma$ 旨 & $\propto$. & $\alpha$ of & ×乐 & બత్రీ & $\therefore$ & a昏 & 四 엉 & $a:$ & & $\propto$ & « ช્ఝ & H & ×孚 \\
\hline & H若 & 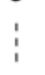 & $H U$ & 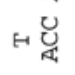 & 日菒 & 的忌 & 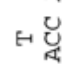 & $H U$ & H歹 & HU & $H \tilde{U}$ & $\vdots$ & E哥 & $+U$ & H䓍 & $\Leftrightarrow U$ \\
\hline & ○秀 & 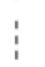 & ט & ט & ○甮 & ○颍 & ○ 영 & ن & ن & 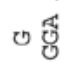 & ن & $\vdots$ & ن & ○ 정 & ن & ن \\
\hline & 导 & $\vdots$ & 幽 & ○ 영 & 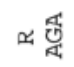 & $\times \frac{0}{4}$ & ه & w苞 & 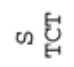 & 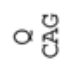 & $\varangle$ \& & $\vdots$ & $\times$ × & هـ & 口尊 & $\times \frac{0}{4}$ \\
\hline & ○ & $\vdots$ & ब తु & ○ 엉 & ○ 정 & ○ 造 & ט 앵 & ৩ 앵 & ○ త్ర & ○ 엉 & ○ 정 & 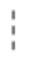 & ن & ○䓵 & o : & 0 잉 \\
\hline & 山葛 & 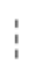 & 袖突 & 的鼠 & 的葸 & 的戒 & 的点 & 山藏 & 的㘀 & 的导 & 山苞 & $\vdots$ & 的葛 & 的葸 & 的菑 & 的鼠 \\
\hline & H旨 & : & & H宏 & 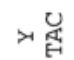 & $\Leftrightarrow$ & 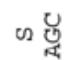 & $x \underset{G}{U}$ & $H \mathcal{U}$ & H旨 & 四 융 & ú & H莚 & $>\breve{y}$ & $H$ & H宗 \\
\hline & 口侮 & 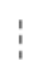 & 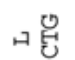 & و & 口. & 蛅 & 븝 & 的萖 & 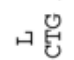 & 口配 & 中兽 & i & 的舅 & 的舅 & ⿰ 幺 & ㅁ뱁 \\
\hline 0 & $ه$ 㨚 & 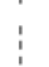 & $\therefore$ 乐 & $\times \frac{\pi}{4}$ & « ర్ర & 後 & $\times$ 雅 & $\therefore \mathbb{4}$ & a歹 & $\because$ 苨 & ×兟 & $\vdots$ & is & z & $\because \frac{\pi}{4}$ & a趙 \\
\hline ర্] & HE⿱宀女甘 & $\vdots$ & 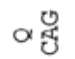 & z导 & ن & $x$ & 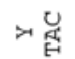 & z & « ర্ & ৩ త্రુ & ن & 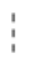 & 山苞 & a ्ठु & ن ठ & $\Rightarrow$ 压 \\
\hline 参 & ○ 정 & 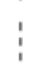 & ن & ○ प्णु & 口 & 山苖 & 口 获 & 的 & « & ט ુु & $3 \stackrel{0}{0}$ & : & ن & ○萼 & $\varangle$ త ర్ర & 的蕠 \\
\hline & $\varangle \widehat{~}$ & $\vdots$ & 口蘦 & « ปું & $H \tilde{G}$ & $\propto$ 숭 & 石䒴 & z曾 & 的葛 & $\alpha \mathscr{H}$ & $\cos$ & $\vdots$ & $\alpha$ क्ष & $\Rightarrow$ 萖 & 口宏 & ن \\
\hline & $\alpha$ 蒫 & $\vdots$ & 的 & 恾 & is & 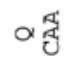 & 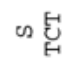 & $\forall \underset{్}{U}$ & ט & 口曾 & 口. 엉 & $\vdots$ & ○ 쟁 & z & $\Leftrightarrow \dot{\widetilde{C}}$ & 《 \\
\hline & z & $\vdots$ & 山苞 & ×承 & ن & $x$ 忎 & 법 & & 我 & $\Rightarrow$ 画 & H若 & : & 四 정 & 口忎 & $z$ & O \\
\hline & u & : & ర্ঠ & 啰 & 乐 & U্ঠ & & & 的葛 & H安 & H串 & $\vdots$ & $\delta$ & O & $\mathrm{H}$ & क苞 \\
\hline & & & & 입 & $\begin{array}{r}\text { 윰 } \\
\text { U }\end{array}$ & & & & $E$ & ${ }_{\mathcal{E}}$ & 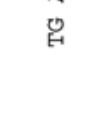 & i & & & & \\
\hline 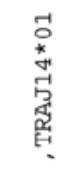 & 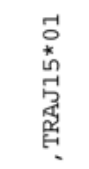 & 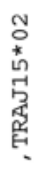 & 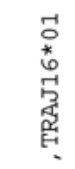 & 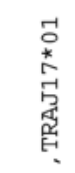 & 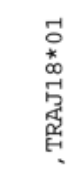 & 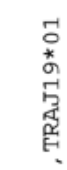 & 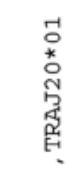 & 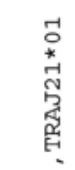 & 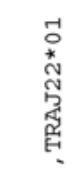 & 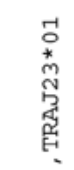 & 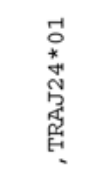 & 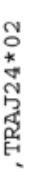 & 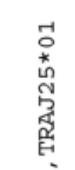 & 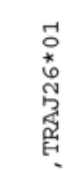 & 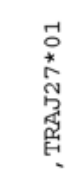 & 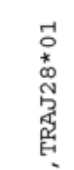 \\
\hline 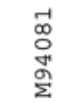 & 怘 & 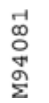 & $\begin{array}{l}\overrightarrow{0} \\
\stackrel{0}{0} \\
\stackrel{0}{\|}\end{array}$ & 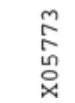 & 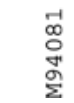 & 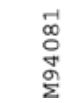 & $\begin{array}{l}\vec{D} \\
0 \\
0 \\
\text { गे }\end{array}$ & 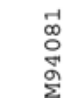 & 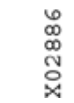 & 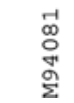 & 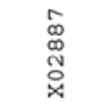 & & $\begin{array}{c}\infty \\
\infty \\
\infty \\
\infty \\
\infty\end{array}$ & 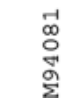 & 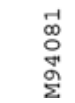 & \\
\hline
\end{tabular}




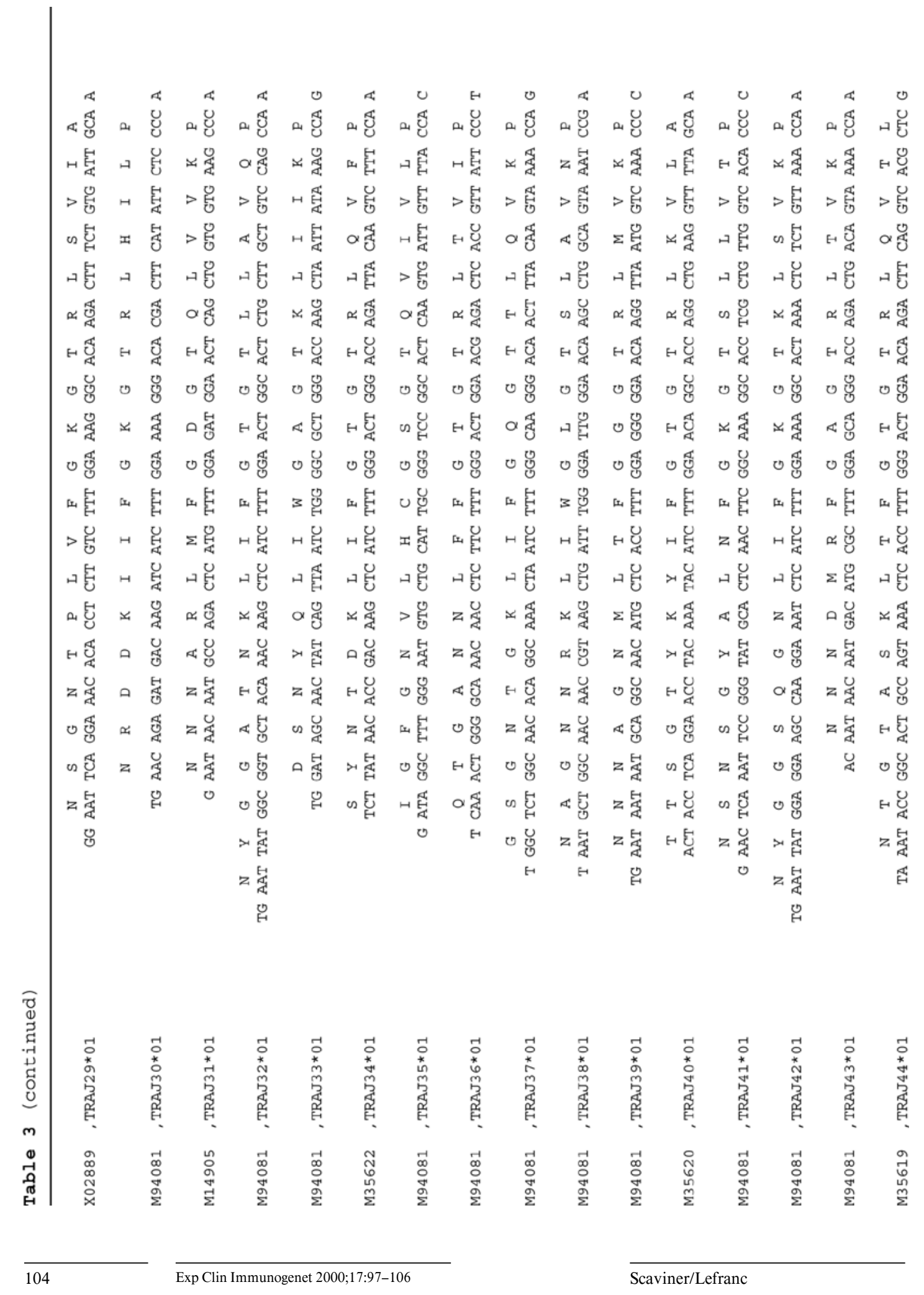


中苟

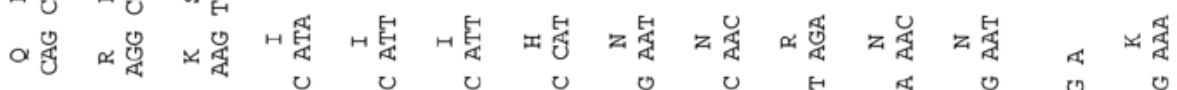

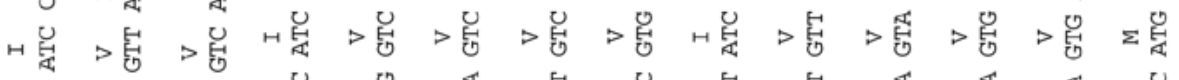

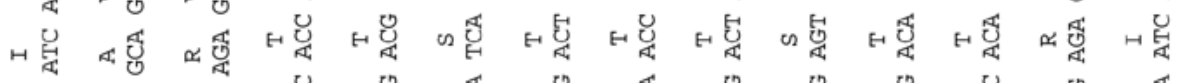

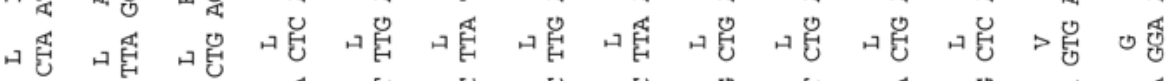

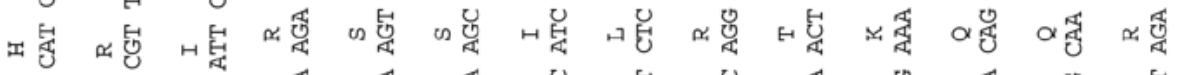

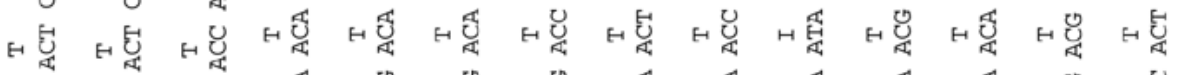

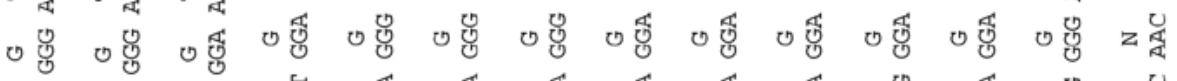

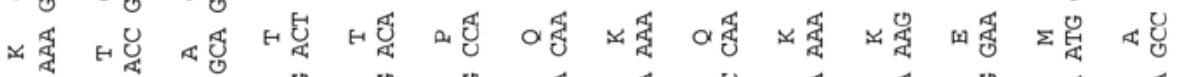

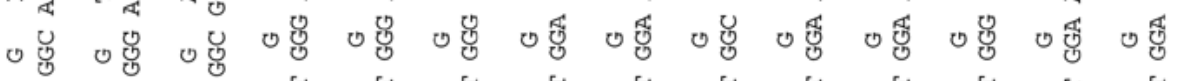

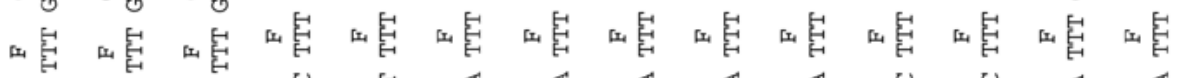

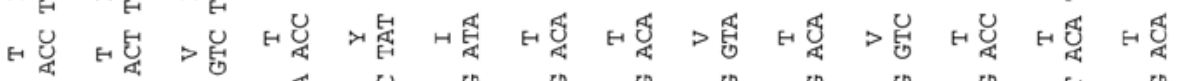

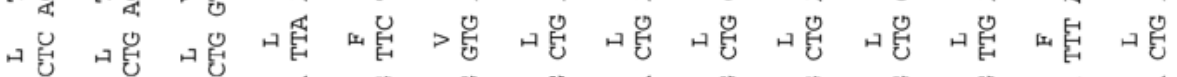

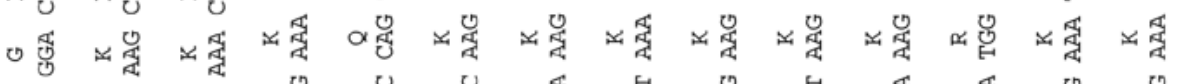

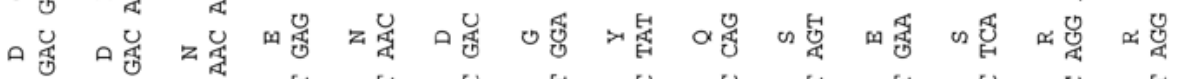

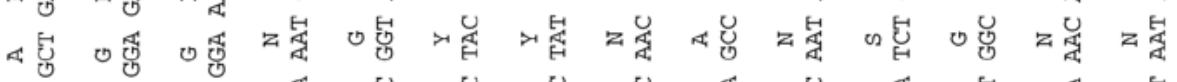

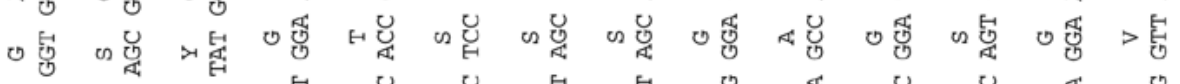

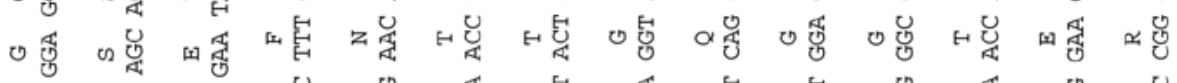

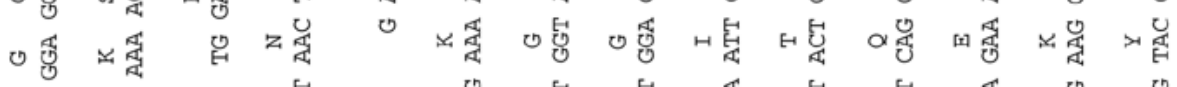

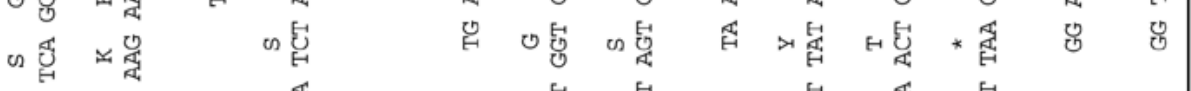

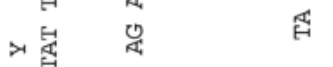

它

《荿察

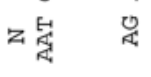

舅

获

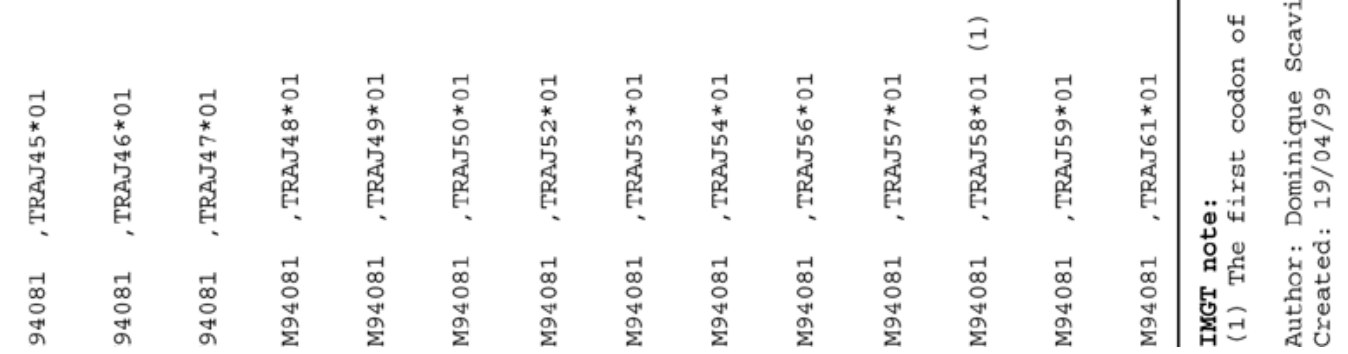

Human TRAJ Genes

Exp Clin Immunogenet 2000;17:97-106 


\section{Acknowledgments}

We thank Valérie Contet for editorial work, Véronique Giudicelli and Gérard Lefranc for helpful discussion. IMGT is funded by the European Union's BIOTECH programme (BIO4CT96-0037), Centre National de la Recherche Scientifique, and Ministère de
l'Education Nationale, de la Recherche et de la Technologie. Subventions have been received from Association pour la Recherche sur le Cancer, Association de la Recherche sur la Polyarthrite, Fondation pour la Recherche Médicale, Ligue Nationale contre le Cancer and Région Languedoc-Roussillon.

\section{References}

1 Lefranc MP: IMGT Locus on focus. Exp Clin Immunogenet 1998;15: $1-7$.

2 Pallarès N, Frippiat JP, Giudicelli V, Lefranc MP: The human immunoglobulin lambda variable (IGLV) genes and joining (IGLJ) segments. Exp Clin Immunogenet 1998;15:818.

3 Barbié V, Lefranc MP: The human immunoglobulin kappa variable (IGKV) genes and joining (IGKJ) segments. Exp Clin Immunogenet 1998;15:171-183.

4 Pallarès $\mathrm{N}$, Lefebvre $\mathrm{S}$, Contet $\mathrm{V}$, Matsuda F, Lefranc MP: The human immunoglobulin heavy variable (IGHV) Genes. Exp Clin Immunogenet 1999;16:36-60.
5 Ruiz M, Pallarès N, Contet V, Barbié V, Lefranc MP: The human immunoglobulin heavy diversity (IGHD) and Joining (IGHJ) segments. Exp Clin Immunogenet 1999;16:173-184.

6 Scaviner D, Barbié V, Ruiz M, Lefranc MP: Protein displays of the human immunoglobulin heavy, kappa and lambda variable and joining regions. Exp Clin Immunogenet 1999;16:234-240.

7 Folch G, Lefranc MP: The human T cell receptor beta variable (TRBV) genes. Exp Clin Immunogenet 2000;17:42-54.
8 Scaviner D, Lefranc MP: The human $\mathrm{T}$ cell receptor alpha variable (TRAV) genes. Exp Clin Immunogenet 2000;17:83-96.

9 Lefranc MP, Giudicelli V, Ginestoux C, Bodmer J, Müller W, Bontrop R, Lemaitre M, Malik A, Barbié V, Chaume D: IMGT, the international ImMunoGeneTics database. Nucleic Acids Res 1999;27: 209-212.

10 Ruiz M, Giudicelli V, Ginestoux C, Stoehr P, Robinson J, Bodmer J, Marsh S, Bontrop R, Lemaitre M, Lefranc G, Chaume D, Lefranc MP: IMGT, the international ImMunoGeneTics database. Nucleic Acids Res 2000;28:219-221. 